\title{
Proanthocyanidin-enriched cranberry extract induces resilient bacterial community dynamics in a gnotobiotic mouse model
}

\author{
Catherine C. Neto ${ }^{1,2, \#, *}$, Benedikt M. Mortzfeld ${ }^{3, \#}$, John R. Turbitt ${ }^{1,2}$, Shakti K. Bhattarai ${ }^{3}$, Vladimir \\ Yeliseyev ${ }^{4}$, Nicholas DiBenedetto ${ }^{4}$, Lynn Bry and Vanni Bucci ${ }^{2,3, *}$ \\ ${ }^{1}$ Department of Chemistry and Biochemistry University of Massachusetts Dartmouth, North Dartmouth, MA. \\ 2 UMass Cranberry Health Research Center, University of Massachusetts Dartmouth, North Dartmouth, MA. \\ ${ }^{3}$ Department of Microbiology and Physiological Systems, University of Massachusetts Medical School, Worcester, MA. \\ ${ }^{4}$ Massachusetts Host-Microbiome Center, Department of Pathology, Brigham and Women's Hospital, Harvard Medical School, \\ Boston MA. \\ \# Equally contributing authors. \\ * Corresponding Authors: \\ Vanni Bucci, 368 Plantation St, Worcester, MA 01605; Phone: 774-455-3854; E-mail: vanni.bucci2@umassmed.edu \\ Catherine C. Neto, 285 Old Westport Road, North Dartmouth, MA 02747; Phone: 508-910-6928; E-mail: cneto@umassd.edu
}

\begin{abstract}
Cranberry consumption has numerous health benefits, with experimental reports showing its anti-inflammatory and anti-tumor properties. Importantly, microbiome research has demonstrated that the gastrointestinal bacterial community modulates host immunity, raising the question of whether the cranberry-derived effect may be related to its ability to modulate the microbiome. Only a few studies have investigated the effect of cranberry products on the microbiome to date. Especially because cranberries are rich in dietary fibers, the extent of microbiome modulation by polyphenols, particularly proanthocyanidins (PACs), remains to be shown. Since previous work has only focused on long-term effects of cranberry extracts, in this study we investigated the effect of a water-soluble, PAC-rich cranberry juice extract (CJE) on the short-term dynamics of a human-derived bacterial community in a gnotobiotic mouse model. CJE characterization revealed a high enrichment in PACs (57\%), the highest ever utilized in a microbiome study. In a 37-day experiment with a ten-day CJE intervention and 14-day recovery phase, we profiled the microbiota via $16 \mathrm{~S}$ rRNA sequencing and applied diverse time-series analytics methods to identify individual bacterial responses. We show that daily administration of CJE induces distinct dynamic patterns in bacterial abundances during and after treatment, before recovering resiliently to pretreatment levels. Specifically, we observed an increase of Akkermansia muciniphila and Clostridium hiranonis at the expense of Bacteroides ovatus after the offset of the selection pressure imposed by the PAC-rich CJE. This demonstrates that termination of an intervention with a cranberry product can induce changes of a magnitude as high as the intervention itself.
\end{abstract}

doi: $10.15698 /$ mic2021.06.752 Received originally: 01.03.2021; In revised form: 16.04.2021, Accepted 22.04.2021, Published 29.04.2021.

Keywords: cranberry extract, polyphenols, proanthocyanidins, microbiome dynamics, gnotobiotic mouse model, microbiome resilience, Akkermansia muciniphila.
Abbreviations:
CJE - cranberry juice extract,
DSS - dextran sodium sulphate, HFHS-high-fat high-sucrose, IBD - inflammatory bowel disease, $P A C$ - proanthocyanidin, UTI- urinary tract infection.

\section{INTRODUCTION}

Cranberry (Vaccinium macrocarpon) is a botanical product used worldwide for the maintenance of a healthy urinary tract. It is consumed in the form of fruit, juice, and other products as part of a diet rich in fibers and polyphenols for the prevention of urinary conditions and diseases of aging including cardiovascular diseases and cancers [1-3]. Cran- berry proanthocyanidins (PACs) and other constituents interact with a wide variety of bacteria, including gut microbes that cause urinary tract infections (UTIs) and other health conditions, by reducing adhesion, biofilm, and coaggregation [4-7]. Persistent gut inflammation, as experienced in inflammatory bowel disease (IBD), has been linked to genetic factors, lifestyle, and dietary habits, in- 
creasing the risk for colon cancer $[8,9]$. Consuming foods high in anti-inflammatory and antioxidant compounds, such as polyphenols or dietary fiber, may therefore provide a preventative strategy to mitigate these conditions and reduce colon cancer risk. Using a DSS-AOM (dextran sodium sulphat - azoxymethane) mouse model of colitisinduced colon tumorigenesis, previous studies by us showed a significant reduction in colon tumors and tissue inflammation in mice fed either whole cranberry powder or cranberry extracts rich in either polyphenols or nonpolyphenol constituents of cranberry [10, 11]. Multiple compounds in cranberries including flavonoids, PACs and triterpenoids have also been reported to reduce tumor cell growth and proliferation, stimulate apoptosis, induce cell cycle arrest, and alter associated signaling processes in cells [12-15].

A significant amount of work has recently demonstrated the role of the gastrointestinal microbiota in modulating host immunity [16]. Seminal studies in animal models have demonstrated that short-chain fatty acids, and in particular butyrate-producing Clostridia species from Clusters IV and $\mathrm{XIVa}$, promote the induction of regulatory T-cells and ameliorate symptoms of colitis $[17,18]$. Furthermore, these bacteria have been associated with the dampening systemic inflammatory response in humans and with the promotion of neurological health of related anti-inflammatory innate immune phenotypes $[19,20]$. Recent work has also shown that specific members of the Bacteroides, Parabacteroides, and Fusobacterium genera robustly induce interferon- $\nu$-producing CD8 T cells in the intestine and enhance therapeutic efficacy of immune checkpoint inhibitors in syngeneic tumor models [21]. Similarly, a recent clinical study demonstrated that patients lacking Akkermansia muciniphila did not respond to PD-1 checkpoint inhibitor immunotherapy [22]. Remarkably oral administration of A. muciniphila was capable of restoring the efficacy of PD-1 blockade in vivo, thus demonstrating the causality of the phenotype and highlighting the importance of this bacterium in modulating anti-cancer immunity [22].

Due to the role that the microbiome has on immune modulation, significant interest is currently placed on understanding the effect of dietary interventions on this system and how diet can be tailored to impact the microbiota and promote health [23-25]. Thanks to this work, it is now established that dietary fibers from plants can promote a healthy and anti-inflammatory microbiome, whereas a diet enriched in animal products has been shown to select for bacteria that have been associated with immune dysregulation and pathogenesis [26].

Interestingly, a few studies have investigated the effect of cranberry extracts on the microbiome and shown that members of the genus Akkermansia, as well as members of the Bifidobacteria and Clostridia order, appear to be positively affected by long-term interventions with cranberry derivatives [27, 28]. Additionally, Bifidobacterium longum subsp. infantis is also associated with the amelioration of symptoms in a DSS-induced gut inflammation mouse model [29]. However, because cranberry fruit averages about $36 \%$ fiber on a dry weight basis, we do not know the extent of microbiome modulation that is due to the sole polyphenols [30]. It is not known, how quickly the microbiome responds to a challenge with polyphenol-rich cranberry extracts, since previous studies only focused on long-term effects. A clearer answer to these questions will provide us with a greater understanding of the role of cranberry polyphenols in modulating gut microbiota dynamics, and how cranberry polyphenol-based dietary interventions could be used to promote gut health in the future.

\section{RESULTS}

\section{Cranberry product composition}

A water-soluble, polyphenol-rich cranberry juice extract (CJE) was chosen for this study, allowing for safe administration via oral gavage to gnotobiotic mice. The major polyphenols in cranberries are poly-flavan-3-ol oligomers, or PACs composed primarily of epicatechin units with two types of linkages, either direct carbon-carbon bonding (B-type) or carbon-carbon bonding with an additional ether linkage between units (A-type). Cranberry fruit ranges widely in soluble PAC content depending on cultivar and other factors [31]. PACs are widely distributed in foods and plant sources, and most contain only B-type linkages. The presence of A-type linkages is characteristic of PACs found in cranberries and other Vaccinium fruits [32]. PACs have long been associated with the urinary health benefits of cranberry, and cranberry juice and extracts have been the subject of multiple clinical trials and other studies, reviewed in [6]. Constituents detected in utilized CJE are summarized in Figure 1A. The total PAC content in the utilized CJE was determined to be $574 \pm 40 \mathrm{mg} / \mathrm{g}$ (57.4\%), using the DMAC method with an authentic cranberry PAC standard. Consistent with previous studies [33], PAC oligomers of up to eight degrees of polymerization and at least one A-type linkage were detected in the $70 \%$ acetone-soluble PAC fraction of CJE by MALDI-TOF MS (Figure 1B, C) Other polyphenols present in CJE detected by HPLC DAD and MALDI-TOF MS analyses include flavonols, primarily quercetin glycosides [34] and anthocyanins, primarily cyanidin and peonidin glycosides (Figure S1, Table S1). The total flavonol content and total anthocyanin content of CJE were $9.6 \pm 0.5 \mathrm{mg} / \mathrm{g}$ and $3.4 \pm 0.3 \mathrm{mg} / \mathrm{g}$, respectively (Figure 1A).

Quantitative ${ }^{1} \mathrm{H}$ NMR analysis found no detectable content of triterpenoids ursolic acid and oleanolic acid, which are typically present in the peel of cranberry fruit and associated with the chemopreventive properties of cranberry $[11,12]$. Whole cranberry fruit contains approximately $10 \mathrm{mg} / \mathrm{g}$ dry weight (1\%) ursolic acid, but due to its low water-solubility, cranberry juice and products derived from juice are much lower in triterpenoid content [13]. No quinic, malic or citric acids were detected, suggesting that smaller organic acids characteristic of cranberry juice were removed by the commercial preparation process (Figure 1A). ${ }^{1} \mathrm{H}$ NMR confirmed the presence of benzoic acid by comparison with an authentic standard, as well as a major derivative of benzoic acid, the glucoside 6-O-benzoyl-Dglucose, which was identified by comparison of aromatic 
A

\begin{tabular}{|c|c|}
\hline Analyte & $\mathrm{mg} / \mathrm{g}$ extract \\
\hline \multicolumn{2}{|l|}{ Polyphenols } \\
\hline Proanthocyanidins (PACs) & $574 \pm 42$ \\
\hline Anthocyanins & $3.4 \pm 0.3$ \\
\hline Flavonol glycosides & $9.6 \pm 0.5$ \\
\hline benzoic acid & 17.6 \\
\hline 6-O-benzoyl-D-glucose & 31 \\
\hline arabinoxyloglucan oligosaccharides & UQ \\
\hline $\begin{array}{l}\text { ursolic acid, oleanic acid, quinic acid } \\
\text { acid were not detected }\end{array}$ & nalic acid and citric \\
\hline
\end{tabular}

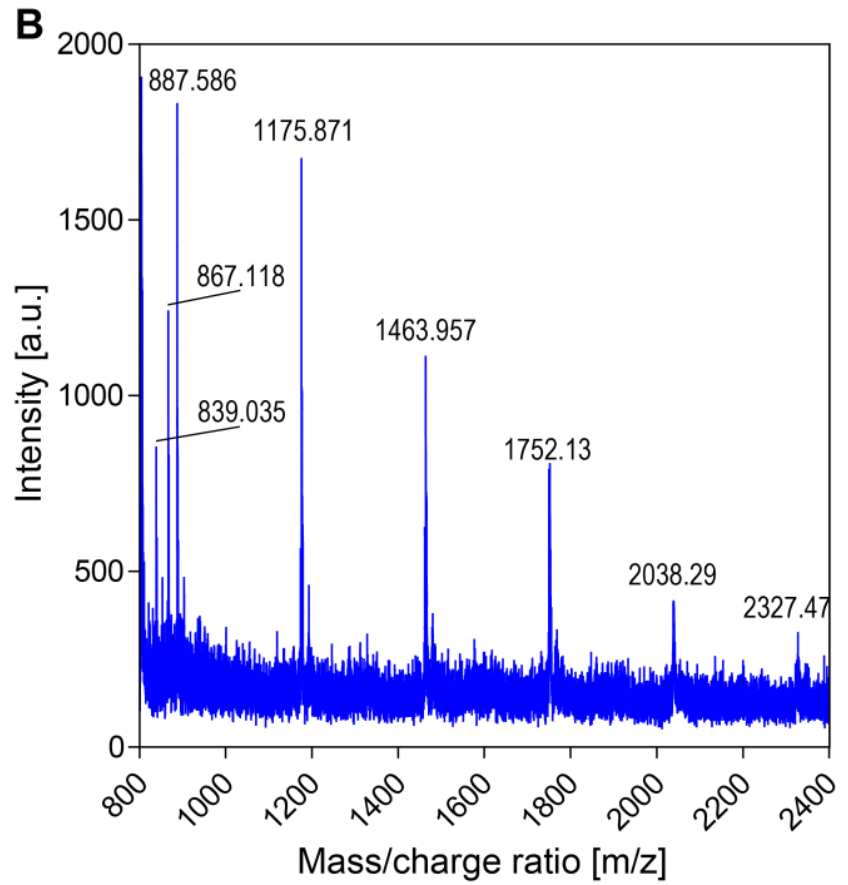

FIGURE 1: Cranberry juice extract composition. (A) Summary of cranberry juice extract components. UQ=Detected in unknown quantity. (B) MALDI-TOF MS spectrum of proanthocyanidin (PAC) fraction from cranberry juice extract, in positive ion mode. (C) Putative identification of the major ion masses in the PAC fraction.

proton signals between $7.4-8.1 \mathrm{ppm}$ and the anomeric proton signal for glucose at $5.6 \mathrm{ppm}$ with those previously reported (Figure S2) [35]. The remaining glucoside signals were obscured by other signals in the $3.5-5.5 \mathrm{ppm}$ region associated with multiple flavonoid glycosides. Based on peak fit integration of aromatic protons for benzoic acid and its glucoside, the CJE contained $30.9 \mathrm{mg}$ 6-O-benzoylD-glucose and $17.6 \mathrm{mg}$ benzoic acid per g dry weight. Thus, CJE contains nearly $5 \%$ free and conjugated benzoic acid. ${ }^{1} \mathrm{H}$ NMR also contained signals between 6.3 and $6.8 \mathrm{ppm}$ characteristic of $\mathrm{p}$-coumaric acid, a major hydroxycinnamic acid in cranberry, however, it appears to be present in very low quantity in CJE.

Multiple ions were detected in the MALDI-TOF MS spectrum of CJE, having masses consistent with previously published data for cranberry oligosaccharides (Figure S3). These included poly-galacturonic acid methyl esters of three and four galacturonic acid units (specifically [M+Na+] at 579 for $\mathrm{uG}^{\mathrm{m} 2} \mathrm{~m} / \mathrm{z}=556$; and $\left[\mathrm{M}+\mathrm{Na}^{+}\right]$at 769 for $\mathrm{uG} 4^{\mathrm{m} 3}$ $\mathrm{m} / \mathrm{z}=746$ ) as reported by Sun and coworkers [36] and a series of larger arabinoxyloglucan oligomers containing between five to nine hexose units and four to eight pentose units. This pattern of oligomer masses is similar to those previously reported in cranberry-derived materials $[37,38]$, but includes larger oligomers, with molecular weights between 1680 and 2532 amu (Table S2). Thus, CJE contains a variety of oligosaccharides. We were unable to quantify oligomer content in CJE due to the lack of appropriate reference standards.

\section{Gut microbiota resilience induced by CJE}

The current literature reports conflicting results on how cranberry-derived compounds affect the microbial gut community. Most of the microbiome modulatory effect has been attributed to high fiber contents of the fruit as well as their high abundance in polyphenols, however, thorough time-dependent in vivo analyses are missing to date, since all previous studies only report analyses through snapshots of selective timepoints [27, 39-41]. While whole cranberry fruit contains approximately $4 \%$ PACs on a dry weight basis $[42,43]$, other studies have utilized moderately enriched extracts $(10 \%)$ to investigate the long-term effect of PACs on the microbiome [40]. We aimed to study the dynamic response of the gut microbiome to a CJE highly enriched in PACs (57\%) throughout the intervention as well as after the treatment. In order to closely monitor the complex microbial dynamics in vivo over several weeks, we chose to utilize a simplified human microbiome consisting of 25 culturable commensal species of human origin (Table S3) [44] Six germ-free C57BL/6J mice were colonized by oral gavage and singly housed under gnotobiotic conditions [45]. After two weeks of colonization, the mice were given $200 \mathrm{mg} / \mathrm{kg}$ body weight $(5 \mathrm{mg}$ ) of CJE daily for ten days, followed by a recovery phase of two weeks (Figure $\mathbf{2 A}$ ).

After first establishment of the gut microbiota at day 9 of the experiment, we found that 15 of the 25 species consistently colonized the murine intestine, as detected by $16 \mathrm{~S}$ rRNA sequencing of the fecal pellets. Before treatment, the microbiota was dominated by Bacteroides ovatus at about $80 \%$ mean relative abundance, a known prominent colonizer of the human gut microflora (Figure S4). When com- 
A

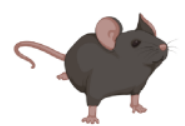

Inoculation with Gnotocomplex 2.0

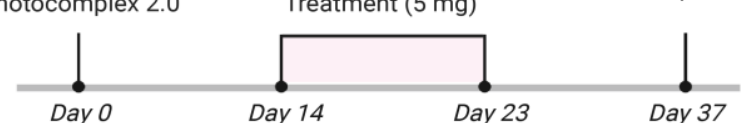

C
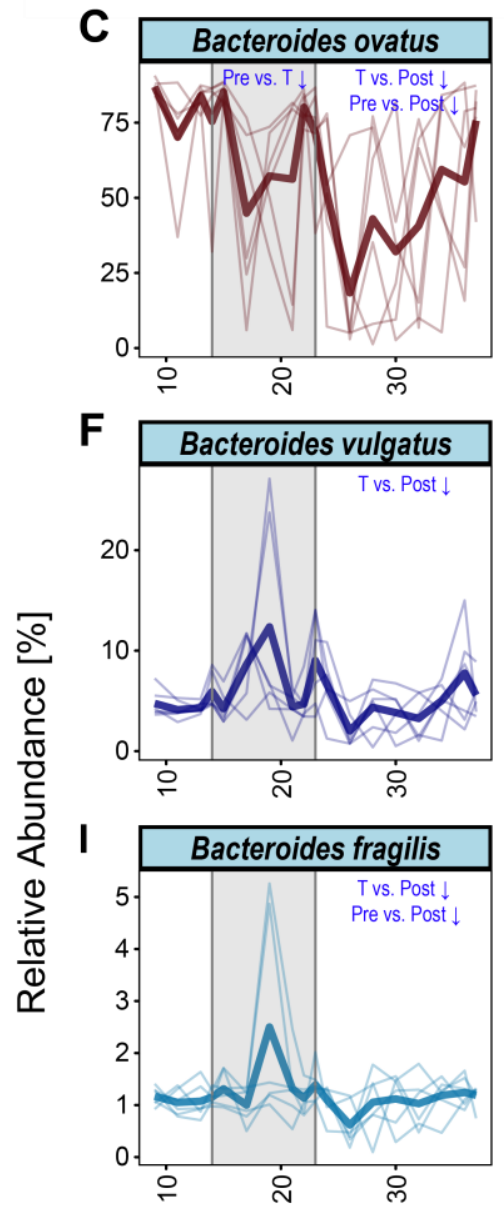

L Parabacteroides distasonis

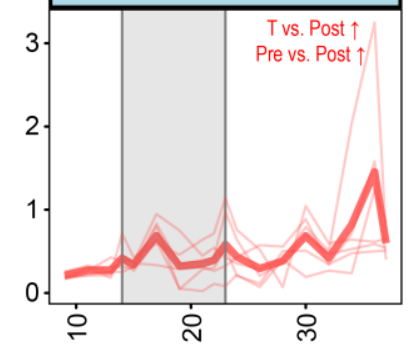

D Akkermansia muciniphila

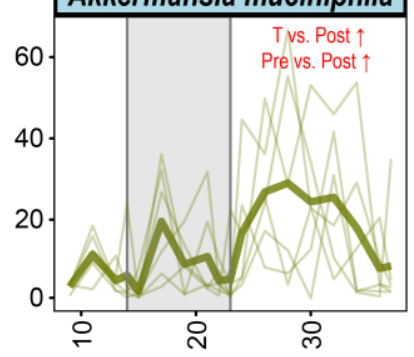

G

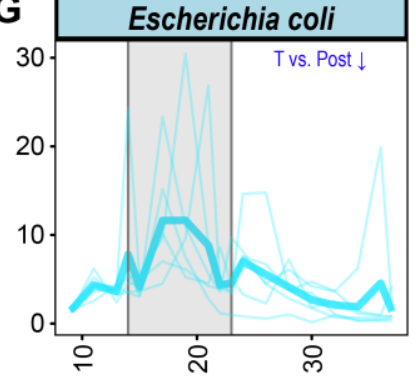

J Clostridium ramosum
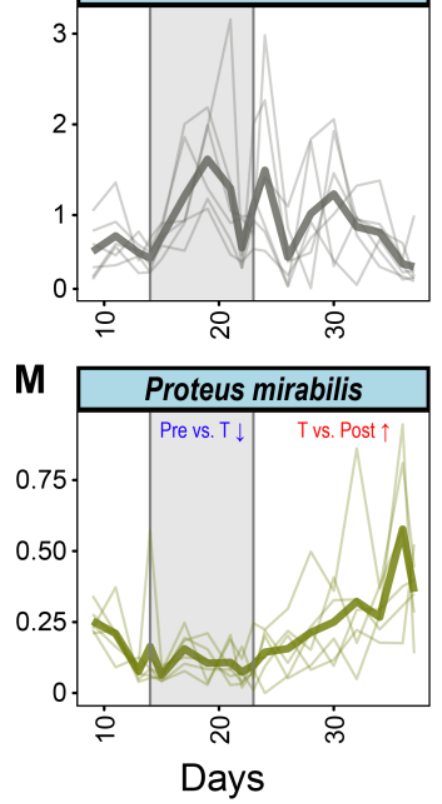

B

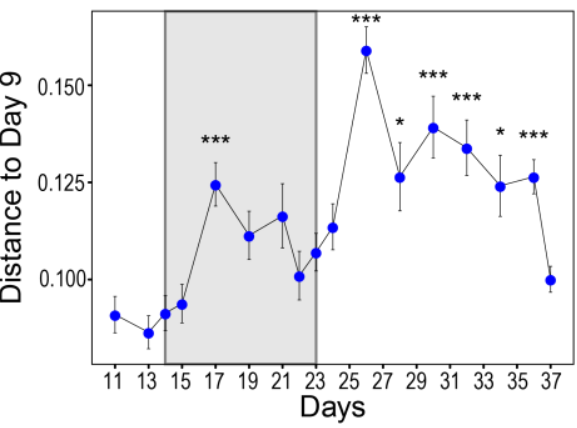

E

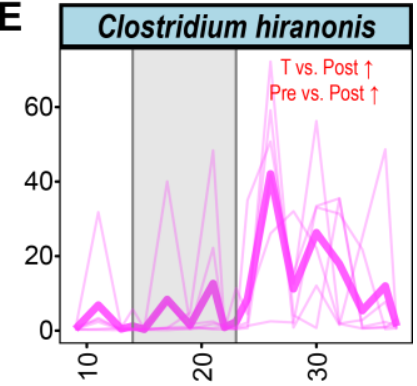

H

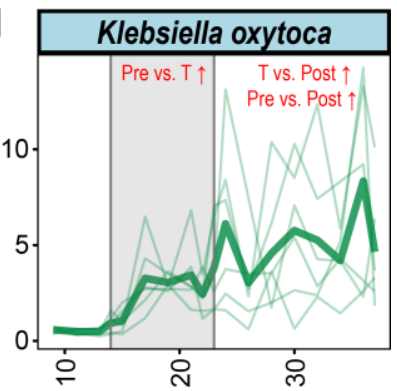

K Lactobacillus reuteri

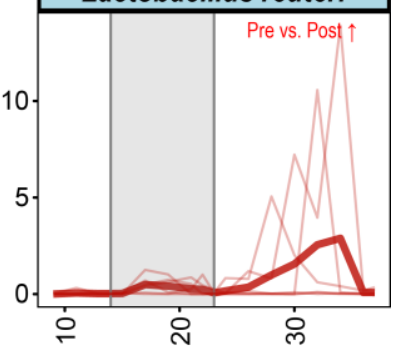

N Enterococcus faecalis

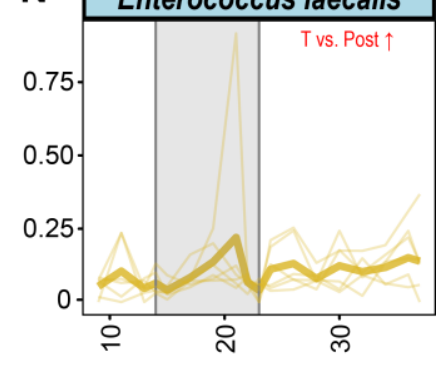

FIGURE 2: CJE treatment modulates the intestinal microbiome in a gnotobiotic mouse model. (A) Schematic of the experimental setup. (B) Bray Curtis distance of the microbial compositions related to the pre-treatment time point day 9. Points and error bars represent mean and standard error of the mean. ${ }^{*}: p \leq 0.05,{ }^{* * *}: p \leq 0.001$. (C-N) Longitudinal depiction of the mean relative abundance throughout the experiment for the $12 / 15$ bacteria that persistently colonized the gnotobiotic mice and were regulated in response to the CJE treatment. Lighter lines show individual replicates. Data for indicated statistical tests are summarized in Figure S5. 
paring the Bray Curtis distances for each time point to the pre-treatment samples taken at day 9 , we found a bimodal dynamic over the course of the experiment driven by the onset and offset of the CJE treatment (Figure 2B). Strikingly, after beginning the treatment with CJE on day 14, we saw a significant increase in the distance to the pre-treatment, indicating major changes in the microbial gut composition. Interestingly, the microbiome recovers towards the end of the treatment around day 22, before offset of the CJE intervention induced another shift in distance at day 26 . Thereafter, the microbiome gradually stabilized resiliently, nearly returning to the pre-treatment level at day 37 . In order to statistically evaluate the changes throughout this longitudinal CJE experiment, we leveraged three tools for statistical analysis (DESeq2, Limma-Voom and ANCOM) to compare the intervals (pre, treatment, post) with one another and report a significant change in relative abundance, when at least $2 / 3$ tests indicated so (Figure S5) [46-48]. We found that CJE treatment itself affected two species of the Enterobacteriaceae family. In particular, Klebsiella oxytoca significantly increased in relative abundance, whereas Proteus mirabilis and the main colonizer $B$. ovatus decreased. As reflected by the distance plot in Figure 2B, a greater number of changes was also observed when comparing abundances after cessation of the treatment (after day 23). Specifically, B. ovatus was found to significantly decrease further in abundance, coinciding with an increase in Clostridium hiranonis and Akkermansia muciniphila (Figure 2, Figure S5), making them the most abundant bacteria after B. ovatus. . oxytoca kept increasing in relative abundance even after the treatment, while $P$. mirabilis returned to pre-treatment levels. Overall, our data suggest that CJE treatment challenged the dominance of $B$. ovatus and promoted expansion of A. muciniphila, $C$. hiranonis and $K$. oxytoca (Figure 2, Figure S5) in a short time frame in this simplified microbial community.

The described analysis relies on predefined intervals, which are set a priori to reflect the treatment boundaries. However, closer examination of the plots in Figure 2 reveals that most of the observed bacterial dynamics may be shorter than the predefined windows. Consequently, none of the applied statistical tools identified E. coli, B. fragilis or $B$. vulgatus to be responding in the treatment window compared to pre-treatment, as their mean relative abundances both spike and recover throughout the ten days of CJE intervention.

In order to unbiasedly define intervals of abundance change in the collected time-series, we applied a change point detection algorithm to the data set $[49,50]$. Briefly, this algorithm infers a position in the time series, where the mean of the relative abundance changes across time intervals. Utilizing this approach, we estimated the intervals of change for each bacterium in each mouse (Figures S6 to Figure S17) as well as for the mean abundance of each bacterial species across multiple mice (Figure 3 ). Interestingly, this approach highlights variability across species in their response to CJE in terms of number of occurrences and locations of change points. Investigating the mean change point plots in Figure $\mathbf{3}$, it becomes apparent that three different dynamics can be observed throughout the treatment. Firstly, there was an early response just after the onset of the CJE treatment, followed by a quick partial to full recovery that, in the majority of cases, was still happening during the CJE intervention. This dynamic can be observed for B. vulgatus, E. coli, B. fragilis, C. ramosum and $E$. faecalis (Figure 3, Table 1). Secondly, we could observe a late response after suspension of the CJE treatment followed by a recovery before the end of the experiment. This dynamic recorded for A. muciniphila, $C$. hiranonis and $L$. reuteri (Figure 3, Table 1 ) may be fueled by the release of the selection pressure imposed by the CJE, allowing for a temporary rearrangement of the microbial community structure post treatment. Lastly, we could observe a set of bacteria that show an early or late response but never experience a recovery in relative abundance, including $B$. ovatus, $K$. oxytoca, $P$. distasonis and $P$. mirabilis. Interestingly, even though the change point algorithm does not detect a recovery for $B$. ovatus in the experimental time frame, the data for the individual mice reveal that a recovery event is detected for $4 / 6$ mice before day 35 (Table 1, Figure S8). Moreover, while the overall pattern looks similar across all mice, the individual responses vary in onset and duration, resulting in a diluted signal and therefore an incomplete recovery in the mean values (Figure $\mathbf{3 A}$ ). Even though the overall resilient bacterial community structure returns to pre-treatment levels at the end of the experiment (Figure 2B), especially the dynamics of the latter bacteria without a recovery demonstrate that an intervention with CJE is able to induce long-term changes in the gut microbiome. Overall, both the treatment with CJE as well as terminating the treatment challenge the dominance of the main colonizer $B$. ovatus, leading to the short-term expansion of other colonizers, including Bacteroides species, Clostridia and Akkermansia. However, in both instances $B$. ovatus showed signs of recovery within two weeks of change.

\section{DISCUSSION}

Cranberry products are consumed around the world for their high nutritional values and antioxidants as well as to prevent UTIs. While it is well established that cranberry derivatives, especially polyphenols, have a modulatory impact on the protective gut microbiome, the mechanisms, by which bacteria influence inflammation-linked processes in gut tissues in the presence of cranberry phytochemicals and their various metabolites, are not established. Other studies of cranberry's effect on the gut microbiota in various mouse models have reported opposite responses of Akkermansia muciniphila in the gut population, in response to treatment with cranberry, linking these effects to the polyphenols [40, 51]. However, polyphenol content and composition in cranberry-derived preparations varies widely depending on source materials and method of preparation, but PACs are typically the major constituent by weight [43]. Anhê and coworkers fed C57BL/6J mice on a high-fat high-sucrose (HFHS) diet incorporating $200 \mathrm{mg}$ per kg body weight of cranberry extract (10\% PACs by weight) for eight weeks. The resulting reduction in insulin resistance and 
intestinal inflammation was associated with a significant increase in A. muciniphila [40]. A related study reported that incorporation of a polyphenol-rich cranberry powder into an HFHS diet restored the functional structure of gut microbiota toward that of mice fed normal chow, with a healthier enterotype lower in Firmicutes and higher in Akkermansiaceae and Coriobacterales [52]. These changes were not induced by the cranberry fiber alone, indicating the polyphenols exert specific effects. In contrast, a study utilizing cranberry powder in a DSS-treated mouse model of gut inflammation found that the A. muciniphila population was boosted significantly by DSS treatment, an effect that could be partially reversed in mice fed cranberry powder for several weeks [51]. While these previous studies focused on long-term microbial effects, reporting single time points after several weeks of treatment, the shortterm effects on the gut microbiome remained unknown. Therefore, we chose a ten-day intervention with a CJE rich
A
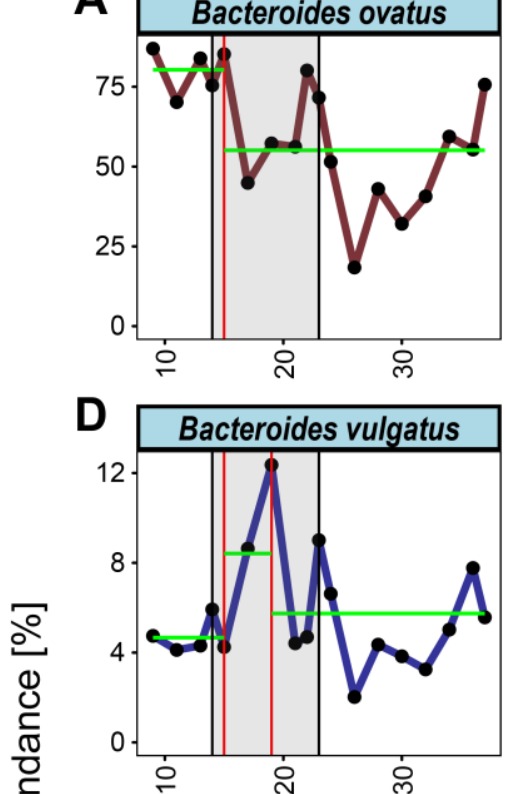

G Bacteroides fragilis
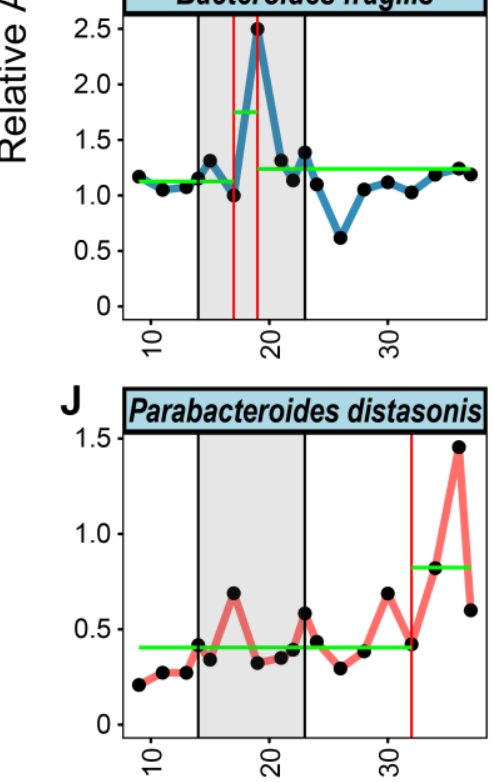
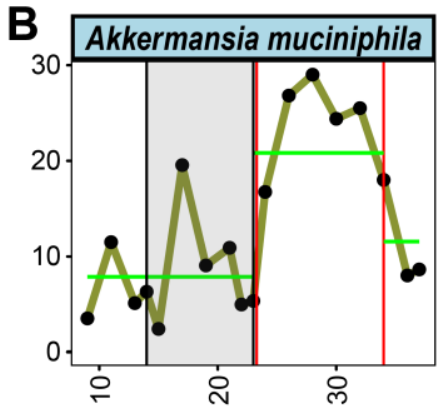

E

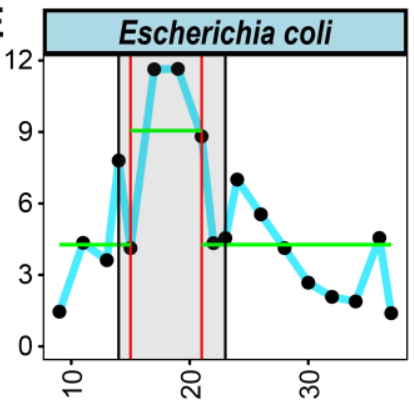

H
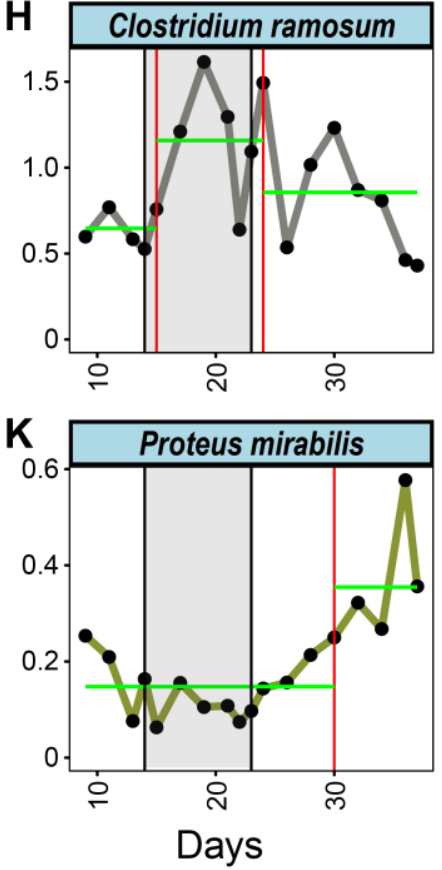

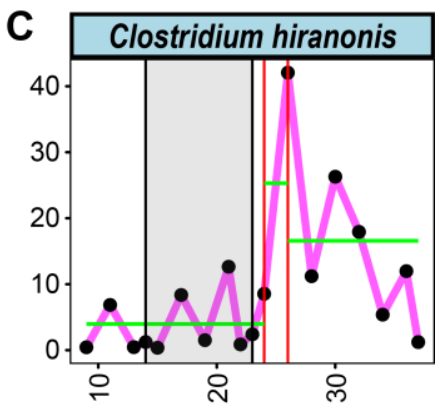

$\mathbf{F}$
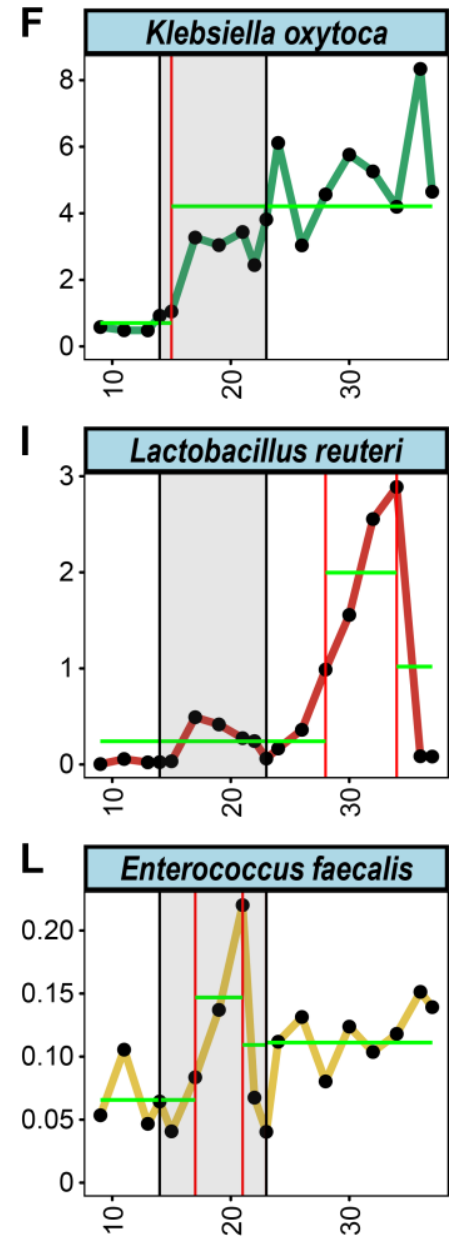

FIGURE 3: Change point analysis of mean relative abundances throughout the experiment. (A-L) Longitudinal depiction of the mean relative abundance throughout the experiment for the $12 / 15$ bacteria that persistently colonized the gnotobiotic mice and were regulated in response to the CJE treatment. Change points are indicated with a red vertical line, segments are indicated with a green horizontal line. Figures for individual replicates are in the Supplementary Figures, a summary of changes and directions can be found in Table 1. 
TABLE 1. Results of changepoint analysis describing the dynamics for every bacterium in each mouse. 'Response' indicates a change from the pre-treatment level, while a 'recovery' marks a subsequent change in the opposite direction. Arrow indicates the direction of the response relative to the pre-treatment level.

\begin{tabular}{|c|c|c|c|c|c|c|c|c|}
\hline & \multicolumn{7}{|c|}{ Response/Recovery } \\
\hline Bacterial species & Strain ID & $\begin{array}{c}\text { Mouse } \\
1\end{array}$ & $\begin{array}{c}\text { Mouse } \\
\text { II }\end{array}$ & $\begin{array}{c}\text { Mouse } \\
\text { III }\end{array}$ & $\begin{array}{c}\text { Mouse } \\
\text { IV }\end{array}$ & $\begin{array}{c}\text { Mouse } \\
\mathrm{V}\end{array}$ & $\begin{array}{c}\text { Mouse } \\
\text { VI }\end{array}$ & Mean \\
\hline Akkermansia muciniphila & DSM 22959 & $2 / 2(\uparrow)$ & $1 / 1(\uparrow)$ & $1 / 1(\uparrow)$ & $1 / 0(\uparrow)$ & $1 / 1(\uparrow)$ & $1 / 1(\uparrow)$ & $1 / 1(\uparrow)$ \\
\hline Bacteroides fragilis & ATCC 25285 & $1 / 1(\uparrow)$ & $1 / 1(\downarrow)$ & $1 / 1(\uparrow)$ & $1 / 1(\downarrow)$ & $1 / 0(\uparrow)$ & $1 / 1(\uparrow)$ & $1 / 1(\uparrow)$ \\
\hline Bacteroides ovatus & ATCC 8483 & $2 / 2(\downarrow)$ & $1 / 1(\downarrow)$ & $1 / 1(\downarrow)$ & $1 / 0(\downarrow)$ & $1 / 0(\downarrow)$ & $2 / 2(\downarrow)$ & $1 / 0(\downarrow)$ \\
\hline Bacteroides vulgatus & ATCC 8482 & $1 / 1(\uparrow)$ & $1 / 1(\uparrow)$ & $1 / 1(\uparrow)$ & $1 / 1(\uparrow)$ & $1 / 1(\uparrow)$ & $1 / 1(\uparrow)$ & $1 / 1(\uparrow)$ \\
\hline Clostridium hiranonis & DSM 13275 & $1 / 1(\uparrow)$ & $1 / 1(\uparrow)$ & $1 / 1(\uparrow)$ & $1 / 1(\uparrow)$ & $1 / 1(\uparrow)$ & $1 / 1(\uparrow)$ & $1 / 1(\uparrow)$ \\
\hline Clostridium ramosum & DSM 1402 & $1 / 1(\uparrow)$ & $1 / 1(\uparrow)$ & $1 / 1(\uparrow)$ & $1 / 1(\uparrow)$ & $1 / 1(\uparrow)$ & $1 / 1(\uparrow)$ & $1 / 1(\uparrow)$ \\
\hline Enterococcus faecalis & ATCC 29200 & $1 / 1(\uparrow)$ & $1 / 0(\uparrow)$ & $1 / 1(\uparrow)$ & $1 / 0(\uparrow)$ & $1 / 0(\uparrow)$ & $1 / 0(\uparrow)$ & $1 / 1(\uparrow)$ \\
\hline Escherichia coli & MG1655 & $1 / 1(\uparrow)$ & $1 / 1(\uparrow)$ & $1 / 1(\uparrow)$ & $1 / 1(\uparrow)$ & $1 / 1(\uparrow)$ & $1 / 0(\downarrow)$ & $1 / 1(\uparrow)$ \\
\hline Klebsiella oxytoca & ATCC 700324 & $1 / 0(\uparrow)$ & $1 / 1(\uparrow)$ & $1 / 0(\uparrow)$ & $1 / 0(\uparrow)$ & $1 / 1(\uparrow)$ & $1 / 0(\uparrow)$ & $1 / 0(\uparrow)$ \\
\hline Lactobacillus reuteri & DSM 20016 & $1 / 1(\uparrow)$ & $1 / 1(\uparrow)$ & $1 / 1(\uparrow)$ & $1 / 1(\uparrow)$ & $1 / 1(\uparrow)$ & $2 / 2(\uparrow)$ & $1 / 1(\uparrow)$ \\
\hline Parabacteroides distasonis & ATCC 8503 & $1 / 1(\uparrow)$ & $1 / 0(\uparrow)$ & $1 / 0(\uparrow)$ & $1 / 0(\uparrow)$ & $1 / 0(\uparrow)$ & $1 / 0(\uparrow)$ & $1 / 0(\uparrow)$ \\
\hline Proteus mirabilis & ATCC 29906 & $1 / 0(\uparrow)$ & $1 / 0(\uparrow)$ & $1 / 0(\uparrow)$ & $1 / 0(\uparrow)$ & $2 / 2(\uparrow)$ & $1 / 0(\uparrow)$ & $1 / 0(\uparrow)$ \\
\hline
\end{tabular}

in polyphenols (57\% PACs) to monitor the immediate community dynamics through time, and after suspension of the treatment. Using a gnotobiotic mouse model, we did not observe a significant increase of $A$. muciniphila during CJE treatment, however, the bacterium was able to flourish at the expense of the main colonizer $B$. ovatus after the treatment, suggesting that it was affected by the PAC-rich CJE during the ten-day intervention.

Cranberry polyphenols have been reported to increase mucin secretion by goblet cells, which helps protect the gut mucous layer and barrier [53]. Akkermansia are mucindegrading bacteria that liberate oligosaccharides from mucin and produce short chain fatty acids [54], which can then be utilized by butyrate-producing bacteria, including commensal Clostridia (clusters XIVa and IV) and other Firmicutes [54]. Interestingly, the expansion of $A$. muciniphila coincides with the expansion of Clostridium hiranonis in our study (Figure 3, Figure S6A, Figure S10A), a cluster XIVa bacterium, whereas Clostridium ramosum (Cluster XVIII) was not significantly affected by the treatment. Commensal Clostridia are strict gram-positive anaerobes that are thought to play important roles in modulating gut homeostasis, maintaining colonocyte health, participation in crosstalk between epithelial and immune cells, and can act as strong inducers of colonic regulatory $T$ cells $\left(T_{\text {regs }}\right)$ [55]. Low abundance of these Clostridia has been linked to inflammatory conditions such as IBD. However, the relationship between $A$. muciniphila and various inflammatory bowel diseases is not completely clear, since overabundance of Akkermansia has been reported to exacerbate the inflammation caused by pathogenic bacteria Salmonella Typhimurium [56, 57].
We would like to point out, that this study utilized a baseline approach, where the pre-treatment community of each mouse serves as the internal for this longitudinal intervention $[19,58]$. While it is unlikely that the treatment with water alone would induce shifts in the gastrointestinal community, we cannot rule out a minor effect through increased stress levels by the daily gavage. Moreover, it is important to note that this and previous studies report relative bacterial abundances without information of actual biomass in the gastrointestinal tract. Therefore, it is possible that certain bacterial species grow in absolute abundance in response to the environmental change, while the main colonizer $B$. ovatus stays unaffected. Nevertheless, it is striking to observe that the mucin-degrading bacterium A. muciniphila appears to be kept in check during the CJE treatment, even though it has been shown that PACrelated goblet cell density and mucus production in the ileum increase within a few days [53]. This suggests that A. muciniphila is susceptible to high concentrations of PACs, but may be able to expand in the community after the treatment, potentially by degrading the accumulated mucin layer and accompanied by butyrate-producing Clostridium hiranonis. However, more detailed longitudinal studies on the impact of cranberry phytochemicals are needed to unravel the mechanisms by which bacteria influence inflammation-linked processes in intestinal tissues and how they manifest in long-term interventions.

In summary our study shows for the first time in a narrowly sampled longitudinal dataset, how a PAC-rich CJE induces community-wide shifts in the intestinal microbiome. Moreover, we are the first to demonstrate that termination of an intervention with a cranberry product induces changes of a magnitude at least as high as the inter- 
vention itself. Both intervals (treatment and post) highlight the strong resilience of the gut microbiome, which was able to recover close to pre-treatment levels within two weeks. While the dominance of $B$. ovatus is mainly challenged by other Bacteroides species, Clostridium ramosum and Escherichia coli after the onset of the treatment, Akkermansia muciniphila and Clostridium hiranonis flourish after offset of the selection pressure imposed by the polyphenol-rich cranberry extract.

\section{MATERIALS AND METHODS}

\section{Cranberry materials and reagents used in characterization}

A food-grade, water-soluble, sterile cranberry-juice derived powder in capsule form (CJE) was donated by Amy Howell of Rutgers University (Ellura ${ }^{\circledR}$, Trophikos, Inc.). The powder is standardized by the manufacturer to contain at least $36 \mathrm{mg}$ of PACs per $240 \mathrm{mg}$ capsule. The capsules were stored at $-20^{\circ} \mathrm{C}$ and in the dark until use. Commercial reagents and standards for analysis were purchased from the following suppliers: Deuterated Dimethylsulfoxide (DMSO- $d_{6}, 99.9 \%$ ) and 4,4dimethyl-4-silapentane-1-sulfonic acid (Cambridge Isotope Laboratories, Andover, MA; N,Ndimethylaminocinnamaldehyde (DMAC), ursolic acid, oleanolic acid (Sigma-Aldrich, St. Louis, MO); malic acid (Eastman Chemicals, Kingsport, TN); citric acid (J.T Baker, Phillipsburg, NJ); quercetin-3-O-galactoside or hyperoside (Chromadex, Irvine, CA); procyanidin-A2 (Indofine Inc., Hillsborough, NJ; quinic acid (Supelco, Bellefonte, PA); cyanidin-3-O-galactoside and peonidin-3-O-galactoside (Extrasynthese, Genay, France).

\section{Total proanthocyanidin determination}

The polyphenol content of the CJE was determined using established methods. Briefly, total PAC content was determined using a modification [59] of the industry standard microplate BL-DMAC assay [60]. An isolated whole fruit cranberry PAC fraction prepared as described previously [33] was used as the standard for the DMAC method, and absorbance measurements were obtained using a microplate reader (Molecular Devices SpectraMax M5, SoftMax Pro V5) as described in [42].

\section{PAC characterization}

PACs were isolated from the fraction for further characterization of oligomers by MALDI-TOF MS (Matrix-Assisted Laser Desorption-Ionization - Time-Of-Flight Mass Spectrometry) using methods established previously.[33] Briefly, free sugars were removed from CJE by chromatography on Diaion-HP20, washing with distilled water, then eluting the polyphenols and oligomers using methanol followed by acetone. The eluate was subjected to further chromatography on Sephadex-LH2O, eluting with 70:30 methanol/water to remove any residual sugars, phenolic acids and flavonoids, followed by elution of proanthocyanidins using 70:30 acetone/water, rotary evaporation and lyophilization. MALDI-TOF MS analysis was performed by Dr. Stephen Eyles at the University of Massachusetts Amherst Mass Spectrometry Facility using a Bruker Daltonics Omniflex MALDI-TOF mass spectrometer. Data acquisition was carried out in positive ion reflectron mode with 0.1 $\mathrm{mM} \mathrm{Csl}, 0.1 \%$ TFA and $50 \mathrm{mM}$ dihydroxybenzoic acid included in the matrix.

\section{HPLC-DAD analysis}

CJE was analyzed for flavonoid composition using HPLC. Identification and quantitation of anthocyanins and flavonol glycosides was performed via reversed-phase HPLC-DAD using a Waters HPLC binary system with 515 pumps coupled with a Waters 996 photodiode array detector and Waters Millenium32 software, as described previously.[42]. Briefly, analyses employed a Waters Atlantis C18 column ( $100 \AA$, $3 \mu \mathrm{m}, 3.9 \mathrm{~mm}$ $x 150 \mathrm{~mm}$ ) and gradient elution at a flow rate of $0.9 \mathrm{~mL} / \mathrm{min}$ with mobile phases consisting of 99.5:0.5 (v/v) water:phosphoric acid (A) and 50:48.5:1:0.5 (v/v/v/v) water:acetonitrile:acetic acid:phosphoric acid (B) according to a published gradient scheme as in [61]. Flavonol glycosides were detected at a wavelength of $355 \mathrm{~nm}$ and quantified based on a quercetin-3-0-glycoside standard; anthocyanins were detected at $520 \mathrm{~nm}$ and quantified based on cyanidin-3-Ogalactoside and peonidin-3-0-galactoside standards as previously described [11].

\section{${ }^{1} \mathrm{H}$ NMR analysis}

A qualitative profile of CJE was generated, and quantitative NMR to determine several non-polyphenol metabolites was conducted using a Bruker AVANCE III $400 \mathrm{MHz}$ NMR spectrometer equipped with a $5 \mathrm{~mm}$ BBFO z-gradient probe, as described previously [42]. Briefly, samples were prepared $(\mathrm{n}=5)$ at $75 \mathrm{mg} / \mathrm{mL}$ in DMSO- $\mathrm{d}_{6}$ with 4,4-dimethyl-4silapentane-1-sulfonic acid as a reference standard. ${ }^{1} \mathrm{H}$ NOESY NMR spectra were acquired and processed using TopSpinTM 3.5 and IconNMRTM 5.0.3 as in [42]. Data analysis was performed using AssureNMRTM 2.0 and AMIXTM 3.9.15. Organic acids and triterpenoids were determined by matching signals against a spectral database and quantified using peak fit integration.

\section{Animal study}

The animal study was conducted under an institutionally approved IACUC protocol. To study the dynamics of the microbiota to a polyphenols-rich cranberry extract, we adopted an approach similar to that presented in [62]. Briefly, six male germ-free C57BL/6 mice at eight weeks of age were transferred into individual cages and checked for sterility by plating fecal pellets before the start of the experiment. In order to closely monitor the complex microbial dynamics in vivo over several weeks, we chose a defined human microbiota consisting of 25 human-origin commensal species (GnotoComplex 2.0 flora), usually found in the gastrointestinal tract. This allowed us to study the effect of CJE on human gut commensals in an in vivo gut environment, simplifying the knowledge transfer to a human study. On day 0 the mice were inoculated with GnotoComplex 2.0 flora by oral gavage [44, 45]. After 14 days, a time in which bacterial establishment can be assessed after initial challenge [62], mice were administered daily a dosage of $5 \mathrm{mg}$ ( $200 \mathrm{mg} / \mathrm{kg}$ body weight) via oral gavage $(0.25 \mathrm{~mL}$ of $20 \mathrm{mg} / \mathrm{mL}$ solution) of CJE in sterilized $\mathrm{H}_{2} \mathrm{O}$ for ten days until day 23 of the experiment. The daily dosage was chosen based on a previously published study, in which a similar dosage appears to have been well-tolerated [63]. Fecal samples were collected every two days throughout the course of the experiment, and daily around the beginning and at end of the CJE treatment. Fecal pellets were snapfrozen and stored at $-80^{\circ} \mathrm{C}$ until DNA extraction with the DNeasy Powersoil kit by Qiagen (Hilden, Germany) according to the manufacturer's protocol. 
The variable regions V3 and V4 of the bacterial 16S rRNA gene were amplified according previously described methods using the universal $341 \mathrm{~F}$ and $806 \mathrm{R}$ primers, and sequenced with 300 nt paired-end sequences on the Illumina MiSeq platform [64].

\section{Bioinformatics and computational analyses}

Forward and reverse $16 \mathrm{~S}$ MiSeq-generated amplicon sequencing reads were dereplicated and sequences were inferred using dada2 [65]. Potentially chimeric sequences were removed using consensus-based methods. Resulting amplicon sequencing variants (ASVs) were mapped to the 16S rRNA gene sequence of the Gnotocomplex 2.0 strains and samples with less than 4000 reads were dropped from the analysis. Sequence files were imported into $R$ and merged with a metadata file into a single Phyloseq object. Due to the repeated-sampling nature of the longitudinal dataset (e.g., paired), we applied three commonly used differential abundance testing methods (DESeq2, Limma-Voom and ANCOM) to determine the effect of CJE on each bacterial species abundance (Figure S5) [46-48]. DESeq2 and Limma-Voom are popular differential abundance testing methods originally developed for RNA-Seq data. DESeq2 models the observed counts of taxa using negative binomial distribution and estimates taxon-wise dispersion parametrically. Limma-Voom on the other hand uses log counts normalized by sequencing depth and estimates mean-variance relation non-parametrically at the individual observation level. It can also model sample correlations for repeated measures data. ANCOM doesn't assume any underlying distribution but uses the compositional structure of microbiome data and utilizes additive log ratios (ALR) in a linear model framework to account for multiple covariates both in cross-sectional and longitudinal settings. Significance thresholds for DESeq2 and Limma-Voom are $p \leq 0.05$ and for ANCOM $W>W_{(0.6)}$. Additionally, we analyzed the abundance data using the change point detections algorithm to detect abrupt shifts in relative abundance of species across different time points (http://sia.webpopix.org/changePoints.html) [49, 50]. Detection of change point is based on the changes in means of relative abundance across time intervals. For a given number of segments $(\mathrm{K}), \mathrm{K}-1$ change points are detected using dynamic programming algorithm, which minimizes the cost of segmentation along with reduced time complexity. To obtain an optimal number of segments $\left(2 \leq \mathrm{K} \leq \mathrm{K}_{\max }\right)$, an elbow curve is

\section{REFERENCES}

1. Vasileiou I, Katsargyris A, Theocharis S, and Giaginis C (2013). Current clinical status on the preventive effects of cranberry consumption against urinary tract infections. Nutr Res 33(8): 595-607. doi: 10.1016/j.nutres.2013.05.018

2. Weh KM, Clarke J, and Kresty LA (2016). Cranberries and Cancer: An Update of Preclinical Studies Evaluating the Cancer Inhibitory Potential of Cranberry and Cranberry Derived Constituents. Antioxid Basel Switz 5(3). doi: 10.3390/antiox5030027

3. Fu Z, Liska D, Talan D, and Chung M (2017). Cranberry Reduces the Risk of Urinary Tract Infection Recurrence in Otherwise Healthy Women: A Systematic Review and Meta-Analysis. J Nutr 147(12): 22822288. doi: 10.3945/jn.117.254961

4. Guay DRP (2009). Cranberry and Urinary Tract Infections. Drugs 69(7): 775-807. doi: 10.2165/00003495-200969070-00002 generated using cost of segmentation with respect to the number of segments. A knee point $\left(\mathrm{K}_{\mathrm{opt}}\right)$ with maximum curvature is estimated using the maximum of second derivative which is approximated using central difference (Figures S6B to Figure S17B).

\section{ACKNOWLEDGEMENTS}

The authors wish to acknowledge Amy Howell of Rutgers University for providing the cranberry product, and the support of the Leo and Anne Albert Charitable Trust, the Commonwealth of Massachusetts, Department of Public Health (INTF4005HH2W20081179), and the Harvard Digestive Diseases Center (P30 DK034854). Mass spectral data were obtained at the University of Massachusetts Mass Spectrometry Center and samples were sequenced at the Center for Microbiome Research (CMR) at the University of Massachusetts Medical School. Gnotobiotic colonization studies were performed at the Massachusetts HostMicrobiome Center. Figure 2A was created with BioRender.com.

\section{SUPPLEMENTAL MATERIAL}

All supplemental data for this article are available online at www.microbialcell.com.

\section{CONFLICT OF INTEREST}

The authors declare no competing interests.

\section{COPYRIGHT}

(C) 2021 Neto et al. This is an open-access article released under the terms of the Creative Commons Attribution (CC BY) license, which allows the unrestricted use, distribution, and reproduction in any medium, provided the original author and source are acknowledged.

Please cite this article as: Catherine C. Neto, Benedikt M. Mortzfeld, John R. Turbitt, Shakti K. Bhattarai, Vladimir Yeliseyev, Nicholas DiBenedetto, Lynn Bry and Vanni Bucci (2021). Proanthocyanidin-enriched cranberry extract induces resilient bacterial community dynamics in a gnotobiotic mouse model. Microbial Cell 8(6): 131-142. doi: 10.15698/mic2021.06.752

5. Vostalova J, Vidlar A, Simanek V, Galandakova A, Kosina P, Vacek J, Vrbkova J, Zimmermann BF, Ulrichova J, and Student V (2015). Are High Proanthocyanidins Key to Cranberry Efficacy in the Prevention of Recurrent Urinary Tract Infection? Phytother Res 29(10): 1559-1567. doi: $10.1002 /$ ptr.5427

6. Blumberg JB, Basu A, Krueger CG, Lila MA, Neto CC, Novotny JA, Reed JD, Rodriguez-Mateos A, and Toner CD (2016). Impact of Cranberries on Gut Microbiota and Cardiometabolic Health: Proceedings of the Cranberry Health Research Conference 2015. Adv Nutr Int Rev J 7(4): 759S-770S. doi: 10.3945/an.116.012583

7. Rauf A, Imran M, Abu-Izneid T, lahtisham-Ul-Haq, Patel S, Pan X, Naz S, Sanches Silva A, Saeed F, and Rasul Suleria HA (2019). Proanthocyanidins: A comprehensive review. Biomed Pharmacother 116: 108999. doi: 10.1016/j.biopha.2019.108999 
8. Colorectal Cancer Risk Factors. Am. Cancer Soc. Available at: https://www.cancer.org/cancer/colon-rectal-cancer/causes-risksprevention/risk-factors.html . [Last Accessed: 16.04.2021]

9. Bernstein CN, Blanchard JF, Kliewer E, and Wajda A (2001). Cancer risk in patients with inflammatory bowel disease: a population-based study. Cancer 91(4): 854-862. doi: 10.1002/10970142(20010215)91:4<854::aid-cncr1073>3.0.co;2-z

10. Wu X, Song M, Cai X, Neto C, Tata A, Han Y, Wang Q, Tang Z, and Xiao $H$ (2018). Chemopreventive effects of whole cranberry (Vaccinium macrocarpon) on colitis-associated colon tumorigenesis. Mol Nutr Food Res 62(24): e1800942. doi: 10.1002/mnfr.201800942

11. Wu X, Xue L, Tata A, Song M, Neto CC, and Xiao H (2020). Bioactive Components of Polyphenol-Rich and Non-Polyphenol-Rich Cranberry Fruit Extracts and Their Chemopreventive Effects on Colitis-Associated Colon Cancer. J Agric Food Chem 68(25): 6845-6853. doi: 10.1021/acs.jafc.0c02604

12. Neto CC, Upton, Roy, and Brendler, Thomas (2016). Anticancer Properties of Cranberry. In: Cranberry Fruit Vaccinium Macrocarpon Aiton - Stand. Anal. Qual. Control Ther. American Herbal Pharmacopoiea; pp 57-61.

13. Kondo M, MacKinnon SL, Craft CC, Matchett MD, Hurta RAR, and Neto CC (2011). Ursolic acid and its esters: occurrence in cranberries and other Vaccinium fruit and effects on matrix metalloproteinase activity in DU145 prostate tumor cells: Anti-tumor activity and content of ursolic acid from Vaccinium fruit. J Sci Food Agric 91(5): 789-796. doi: 10.1002/jsfa.4330

14. Déziel B, MacPhee J, Patel K, Catalli A, Kulka M, Neto C, GottschallPass K, and Hurta R (2012). American cranberry (Vaccinium macrocarpon) extract affects human prostate cancer cell growth via cell cycle arrest by modulating expression of cell cycle regulators. Food Funct 3(5): 556. doi: 10.1039/c2fo10145a

15. MacLean MA, Scott BE, Deziel BA, Nunnelley MC, Liberty AM, Gottschall-Pass KT, Neto CC, and Hurta RAR (2011). North American cranberry (Vaccinium macrocarpon) stimulates apoptotic pathways in DU145 human prostate cancer cells in vitro. Nutr Cancer 63(1): 109120. doi: 10.1080/01635581.2010.516876

16. Belkaid $Y$, and Hand TW (2014). Role of the Microbiota in Immunity and Inflammation. Cell 157(1): 121-141. doi: 10.1016/j.cell.2014.03.011

17. Atarashi K, Tanoue T, Oshima K, Suda W, Nagano $Y$, Nishikawa H, Fukuda S, Saito T, Narushima S, Hase K, Kim S, Fritz JV, Wilmes P, Ueha S, Matsushima K, Ohno H, Olle B, Sakaguchi S, Taniguchi T, Morita H, Hattori $M$, and Honda $K$ (2013). Treg induction by a rationally selected mixture of Clostridia strains from the human microbiota. Nature 500(7461): 232-236. doi: 10.1038/nature12331

18. Abdel-Gadir A, Stephen-Victor E, Gerber GK, Noval Rivas M, Wang S, Harb H, Wang L, Li N, Crestani E, Spielman S, Secor W, Biehl H, DiBenedetto N, Dong X, Umetsu DT, Bry L, Rachid R, and Chatila TA (2019). Microbiota therapy acts via a regulatory T cell MyD88/RORyt pathway to suppress food allergy. Nat Med 25(7): 1164-1174. doi: 10.1038/s41591-019-0461-z

19. Wipperman MF, Bhattarai SK, Vorkas CK, Maringati VS, Taur Y, Mathurin L, McAulay K, Vilbrun SC, Francois D, Bean J, Walsh KF, Nathan C, Fitzgerald DW, Glickman MS, and Bucci V (2021). Gastrointestinal microbiota composition predicts peripheral inflammatory state during treatment of human tuberculosis. Nat Commun 12(1): 1141. doi: 10.1038/s41467-021-21475-y

20. Haran JP, Bhattarai SK, Foley SE, Dutta P, Ward DV, Bucci V, and McCormick BA (2019). Alzheimer's Disease Microbiome Is Associated with Dysregulation of the Anti-Inflammatory P-Glycoprotein Pathway. mBio 10(3): e00632-19. doi: 10.1128/mBio.00632-19
21. Tanoue T, Morita S, Plichta DR, Skelly AN, Suda W, Sugiura Y, Narushima S, Vlamakis H, Motoo I, Sugita K, Shiota A, Takeshita K, Yasuma-Mitobe K, Riethmacher D, Kaisho T, Norman JM, Mucida D, Suematsu M, Yaguchi T, Bucci V, Inoue T, Kawakami Y, Olle B, Roberts B, Hattori M, Xavier RJ, Atarashi K, and Honda K (2019). A defined commensal consortium elicits CD8 $T$ cells and anti-cancer immunity. Nature 565(7741): 600-605. doi: 10.1038/s41586-019-0878-z

22. Routy B et al. (2018). Gut microbiome influences efficacy of PD-1based immunotherapy against epithelial tumors. Science 359(6371): 91-97. doi: 10.1126/science.aan3706

23. David LA, Maurice CF, Carmody RN, Gootenberg DB, Button JE, Wolfe BE, Ling AV, Devlin AS, Varma Y, Fischbach MA, Biddinger SB, Dutton RJ, and Turnbaugh PJ (2014). Diet rapidly and reproducibly alters the human gut microbiome. Nature 505(7484): 559-563. doi: $10.1038 /$ nature 12820

24. Yang Q, Liang Q, Balakrishnan B, Belobrajdic DP, Feng Q-J, and Zhang W (2020). Role of Dietary Nutrients in the Modulation of Gut Microbiota: A Narrative Review. Nutrients 12(2): 381 . doi: 10.3390/nu12020381

25. Olednzki B, Bucci V, Cawley C, McCormick B, Ward D, and Maldonado-Contreras A (2020). P073 DIET AS A MICROBIOME-CENTERED THERAPY FOR IBD. Gastroenterology 158(3): S57-S58. doi: 10.1053/j.gastro.2019.11.284

26. Sonnenburg JL, and Bäckhed F (2016). Diet-microbiota interactions as moderators of human metabolism. Nature 535(7610): 56-64. doi: $10.1038 /$ nature18846

27. Anhê FF, Nachbar RT, Varin TV, Vilela V, Dudonné S, Pilon G, Fournier $M$, Lecours $M-A$, Desjardins $Y$, Roy $D$, Levy $E$, and Marette $A$ (2017). A polyphenol-rich cranberry extract reverses insulin resistance and hepatic steatosis independently of body weight loss. Mol Metab 6(12): 1563-1573. doi: 10.1016/j.molmet.2017.10.003

28. Liu J, Hao W, He Z, Kwek E, Zhu H, Ma N, Ma KY, and Chen Z-Y (2021). Blueberry and cranberry anthocyanin extracts reduce bodyweight and modulate gut microbiota in C57BL/ $6 \mathrm{~J}$ mice fed with a high-fat diet. Eur J Nutr. doi: 10.1007/s00394-020-02446-3

29. Nguyen T, Kimble L, Mathison B, and Chew B (2015). Whole Cranberry Powder Promotes Bifidobacterium infantis Attenuation of Disease Activity in Dextran Sulfate Sodium-Induced Colitis in Mice. FASEB J 29(S1): 608.35. doi: 10.1096/fasebj.29.1_supplement.608.35

30. FoodData Central. Available at: https://fdc.nal.usda.gov/fdcapp.html\#/food-details/1102706/nutrients [Last Accessed 20.02.2021].

31. Carpenter JL, Caruso FL, Tata A, Vorsa N, and Neto CC (2014). Variation in proanthocyanidin content and composition among commonly grown North American cranberry cultivars ( Vaccinium macrocarpon ): Proanthocyanidin content of cranberries. J Sci Food Agric 94(13): 2738-2745. doi: 10.1002/jsfa.6618

32. Reed JD, Krueger CG, and Vestling MM (2005). MALDI-TOF mass spectrometry of oligomeric food polyphenols. Phytochemistry 66(18): 2248-2263. doi: 10.1016/j.phytochem.2005.05.015

33. Patel KD, Scarano FJ, Kondo M, Hurta RAR, and Neto CC (2011). Proanthocyanidin-rich extracts from cranberry fruit (Vaccinium macrocarpon Ait.) selectively inhibit the growth of human pathogenic fungi Candida spp. and Cryptococcus neoformans. J Agric Food Chem 59(24): 12864-12873. doi: 10.1021/jf2035466

34. Turbitt JR (2021). Analysis of Commercially Available Cranberry (Vaccinium macrocarpon) Supplements and Their Effect on Common Gut Microbes. Doctoral Dissertation, University of Massachusetts Dartmouth.

35. He X, and Liu RH (2006). Cranberry Phytochemicals: Isolation, Structure Elucidation, and Their Antiproliferative and Antioxidant 
Activities. J Agric Food Chem 54(19): 7069-7074. doi: 10.1021/jf061058I

36. Sun J, Deering RW, Peng Z, Najia L, Khoo C, Cohen PS, Seeram NP, and Rowley DC (2019). Pectic Oligosaccharides from Cranberry Prevent Quiescence and Persistence in the Uropathogenic Escherichia coli CFT073. Sci Rep 9(1): 19590. doi: 10.1038/s41598-019-56005-w

37. Hotchkiss AT, Nuñez A, Strahan GD, Chau HK, White AK, Marais JPJ, Hom K, Vakkalanka MS, Di R, Yam KL, and Khoo C (2015). Cranberry Xyloglucan Structure and Inhibition of Escherichia coli Adhesion to Epithelial Cells. J Agric Food Chem 63(23): 5622-5633. doi: 10.1021/acs.jafc.5b00730

38. Neto CC, Penndorf KA, Feldman M, Meron-Sudai S, Zakay-Rones Z, Steinberg D, Fridman M, Kashman Y, Ginsburg I, Ofek I, and Weiss EI (2017). Characterization of non-dialyzable constituents from cranberry juice that inhibit adhesion, co-aggregation and biofilm formation by oral bacteria. Food Funct 8(5): 1955-1965. doi: 10.1039/C7FO00109F

39. Straub TJ, Chou W-C, Manson AL, Schreiber HL, Walker BJ, Desjardins CA, Chapman SB, Kaspar KL, Kahsai OJ, Traylor E, Dodson KW, Hullar MAJ, Hultgren SJ, Khoo C, and Earl AM (2021). Limited effects of long-term daily cranberry consumption on the gut microbiome in a placebo-controlled study of women with recurrent urinary tract infections. BMC Microbiol 21(1): 53. doi: 10.1186/s12866-02102106-4

40. Anhê FF, Roy D, Pilon G, Dudonné S, Matamoros S, Varin TV, Garofalo C, Moine Q, Desjardins Y, Levy E, and Marette A (2015). A polyphenol-rich cranberry extract protects from diet-induced obesity, insulin resistance and intestinal inflammation in association with increased Akkermansia spp. population in the gut microbiota of mice. Gut 64(6): 872-883. doi: 10.1136/gutjnl-2014-307142

41. Rodríguez-Morató J, Matthan NR, Liu J, de la Torre R, and Chen CYO (2018). Cranberries attenuate animal-based diet-induced changes in microbiota composition and functionality: a randomized crossover controlled feeding trial. J Nutr Biochem 62: 76-86. doi: 10.1016/j.jnutbio.2018.08.019

42. Turbitt JR, Colson KL, Killday KB, Milstead A, and Neto CC (2020). Application of $1 \mathrm{H}-\mathrm{NMR}$-based metabolomics to the analysis of cranberry (Vaccinium macrocarpon) supplements. Phytochem Anal PCA 31(1): 68-80. doi: 10.1002/pca.2867

43. Bhagwat S, Haytowitz DB (2015). USDA Database for the Proanthocyanidin Content of Selected Foods, Release 2 (2015). Nutrient Data Laboratory, Beltsville Human Nutrition Research Center, ARS, USDA. doi: 10.15482/USDA.ADC/1324621

44. Riglar DT, Giessen TW, Baym M, Kerns SJ, Niederhuber MJ, Bronson RT, Kotula JW, Gerber GK, Way JC, and Silver PA (2017). Engineered bacteria can function in the mammalian gut long-term as live diagnostics of inflammation. Nat Biotechnol 35(7): 653-658. doi: $10.1038 / \mathrm{nbt} .3879$

45. Lavin R, DiBenedetto N, Yeliseyev V, Delaney M, and Bry L (2018). Gnotobiotic and Conventional Mouse Systems to Support Microbiota Based Studies. Curr Protoc Immunol 121(1): e48. doi: 10.1002/cpim.48

46. Love MI, Huber W, and Anders S (2014). Moderated estimation of fold change and dispersion for RNA-seq data with DESeq2. Genome Biol 15(12): 550. doi: 10.1186/s13059-014-0550-8

47. Law CW, Chen Y, Shi W, and Smyth GK (2014). voom: precision weights unlock linear model analysis tools for RNA-seq read counts. Genome Biol 15(2): R29. doi: 10.1186/gb-2014-15-2-r29

48. Mandal S, Treuren WV, White RA, Eggesb $\varnothing$ M, Knight R, and Peddada SD (2015). Analysis of composition of microbiomes: a novel method for studying microbial composition. Microb Ecol Health Dis 26(1): 27663. doi: 10.3402/mehd.v26.27663
49. Killick $R$, and Eckley IA (2014). changepoint: An $R$ Package for Changepoint Analysis. J Stat Softw 58(3). doi: 10.18637/jss.v058.i03

50. Lavielle M (2005). Using penalized contrasts for the change-point problem. Signal Process 85(8): 1501-1510. doi: 10.1016/j.sigpro.2005.01.012

51. Cai X, Han Y, Gu M, Song M, Wu X, Li Z, Li F, Goulette T, and Xiao H (2019). Dietary cranberry suppressed colonic inflammation and alleviated gut microbiota dysbiosis in dextran sodium sulfate-treated mice. Food Funct 10(10): 6331-6341. doi: 10.1039/C9FO01537J

52. Rodríguez-Daza M-C, Roquim $M$, Dudonné S, Pilon G, Levy $E$, Marette A, Roy D, and Desjardins Y (2020). Berry Polyphenols and Fibers Modulate Distinct Microbial Metabolic Functions and Gut Microbiota Enterotype-Like Clustering in Obese Mice. Front Microbiol 11: 2032. doi: 10.3389/fmicb.2020.02032

53. Pierre JF, Heneghan AF, Feliciano RP, Shanmuganayagam D, Roenneburg DA, Krueger CG, Reed JD, and Kudsk KA (2013). Cranberry Proanthocyanidins Improve the Gut Mucous Layer Morphology and Function in Mice Receiving Elemental Enteral Nutrition. J Parenter Enter Nutr 37(3): 401-409. doi: 10.1177/0148607112463076

54. Lordan C, Thapa D, Ross RP, and Cotter PD (2020). Potential for enriching next-generation health-promoting gut bacteria through prebiotics and other dietary components. Gut Microbes 11(1): 1-20. doi: 10.1080/19490976.2019.1613124

55. Lopetuso LR, Scaldaferri F, Petito V, and Gasbarrini A (2013). Commensal Clostridia: leading players in the maintenance of gut homeostasis. Gut Pathog 5(1): 23. doi: 10.1186/1757-4749-5-23

56. Anhê FF, Pilon G, Roy D, Desjardins Y, Levy E, and Marette A (2016). Triggering Akkermansia with dietary polyphenols: A new weapon to combat the metabolic syndrome? Gut Microbes 7(2): 146-153. doi: 10.1080/19490976.2016.1142036

57. Ganesh BP, Klopfleisch R, Loh G, and Blaut M (2013). Commensal Akkermansia muciniphila Exacerbates Gut Inflammation in Salmonella Typhimurium-Infected Gnotobiotic Mice. PLOS ONE 8(9): e74963. doi: 10.1371/journal.pone.0074963

58. Dethlefsen L, and Relman DA (2011). Incomplete recovery and individualized responses of the human distal gut microbiota to repeated antibiotic perturbation. Proc Natl Acad Sci U S A 108 (Suppl 1): 4554-4561. doi: 10.1073/pnas.1000087107

59. Feliciano RP, Shea MP, Shanmuganayagam D, Krueger CG, Howell $A B$, and Reed JD (2012). Comparison of isolated cranberry (Vaccinium macrocarpon Ait.) proanthocyanidins to catechin and procyanidins A2 and B2 for use as standards in the 4-(dimethylamino)cinnamaldehyde assay. J Agric Food Chem 60(18): 4578-4585. doi: 10.1021/jf3007213

60. Prior RL, Fan E, Ji H, Howell A, Nio C, Payne MJ, and Reed J (2010). Multi-laboratory validation of a standard method for quantifying proanthocyanidins in cranberry powders. J Sci Food Agric 90(9): 14731478. doi: 10.1002/jsfa.3966

61. Brown PN, and Shipley PR (2011). Determination of Anthocyanins in Cranberry Fruit and Cranberry Fruit Products by High-Performance Liquid Chromatography with Ultraviolet Detection: Single-Laboratory Validation. J Aoac Int 94(2): 459-466. doi: 10.1093/jaoac/94.2.459

62. Bucci V, Tzen B, Li N, Simmons M, Tanoue T, Bogart E, Deng L, Yeliseyev V, Delaney ML, Liu Q, Olle B, Stein RR, Honda K, Bry L, and Gerber GK (2016). MDSINE: Microbial Dynamical Systems INference Engine for microbiome time-series analyses. Genome Biol 17(1): 121. doi: 10.1186/s13059-016-0980-6

63. Singh DP, Singh S, Bijalwan V, Kumar V, Khare P, Baboota RK, Singh $P$, Boparai RK, Singh J, Kondepudi KK, Chopra K, and Bishnoi M (2018). Co-supplementation of isomalto-oligosaccharides potentiates metabolic health benefits of polyphenol-rich cranberry extract in high fat 
diet-fed mice via enhanced gut butyrate production. Eur J Nutr 57(8): 2897-2911. doi: 10.1007/s00394-017-1561-5

64. Kozich JJ, Westcott SL, Baxter NT, Highlander SK, and Schloss PD (2013). Development of a Dual-Index Sequencing Strategy and Curation Pipeline for Analyzing Amplicon Sequence Data on the MiSeq
Illumina Sequencing Platform. Appl Environ Microbiol 79(17): 51125120. doi: 10.1128/AEM.01043-13

65. Callahan BJ, McMurdie PJ, Rosen MJ, Han AW, Johnson AJA, and Holmes SP (2016). DADA2: High-resolution sample inference from Illumina amplicon data. Nat Methods 13(7): 581-583. doi: 10.1038/nmeth.3869 